\title{
Software for the Fermilab FASTBUS Smart Crate Controller ${ }^{1}$
}

\author{
David Berg, Eileen Berman, Bryan MacKinnon, Tom Nicinski,Gene Oleynik, Don \\ Petravick, Ruth Pordes, Gary Sergey, David Slimmer, Jonathan Streets ${ }^{2}$ \\ Online and Data Acquisition Software Groups \\ Online Support Department \\ Fermi National Accelerator Laboratory \\ Batavia, Illinois 60510 \\ Wolfgang Kowald \\ Duke University
}

\begin{abstract}
We report on software developed in support of the Fermilab FASTBUS Smart Crate Controller. This software includes a full suite of diagnostics, support for FASTBUS Standard Routines, and extended software to allow communication over the RS-232 and Ethernet ports. The communication software supports remote procedure call execution from a host VAX or Unix system. The software supported on the FSCC forms part of the PAN-DA software system which supports the functions of front end readout controllers and event builders in multiprocessor, multilevel, distributed data acquisition systems.
\end{abstract}

\section{INTRODUCTION}

The Fermilab FASTBUS Smart Crate Controller (FSCC) is an intelligent readout controller intended to fill the void which existed between general purpose masters and the LeCroy 1821 FASTBUS Readout Controller. Besides being able to perform high speed sequencer readout, the FSCC is capable of lower rate, but more flexible, readout controlled by a Motorola 68020 processor. The data stream can be accessed by the processor to perform monitoring and/or analysis tasks. A rich programming environment is provided by a variety of on-board programmable components: a memory mapped FASTBUS interface, Ethemet interface, serial line interface, parallel port interfaces, programmable interrupt handler, and programmable interval timers.

The FSCC is currently in use by two Fermilab experiments: E-771 and E-791. E-791 has successfully taken seven Terabytes of event data with the FSCC during the last fixed target running period. They used 10 FSCCs at their full rate to read events into VDAS buffers in VME, where they were processed and written to $8 \mathrm{~mm}$ tape. The FSCC is an

\footnotetext{
${ }_{1}$ Sponsored by DOE contract No. DE-AC02-76CH03000

${ }^{2}$ The authors would like to thank the Online Support and Data Acquisition Electronics Departments
}

integral component of the Silicon Strip Detector readout system under development at Fermilab which will be used by E-771 during the next fixed target run.

The Fermilab Online Support Department provides software support for the FSCC, as a component of the PANDA data acquisition system [1], in following areas:

- System level software:

- A multi-tasking real-time operating kernel, pSOS, and board level debugger, pROBE

- Cross development tools: C compiler, linker, etc.

- Embedded systems board environment

- ROM resident applications (e.g. Ethernet downloader)

- Automatic downloading at boot time

- ROM resident FSCC diagnostic software

- Communications software:

- Serial Port driver

- Ethernet driver and "unreliable" datagram communications

- Reliable datagram communications

- Remote Procedure Execution over the Serial line or Ethernet

- FASTBUS Standard Routines

\section{FSCC FUNCTIONALITY}

Figure 1 is a functional diagram of the FSCC. A companion paper [2] presented at this conference and an FSCC design document [3] contain detailed descriptions of the hardware aspects of the FSCC. Only a brief summary is presented here for background. 
FSCC Functional Diagram

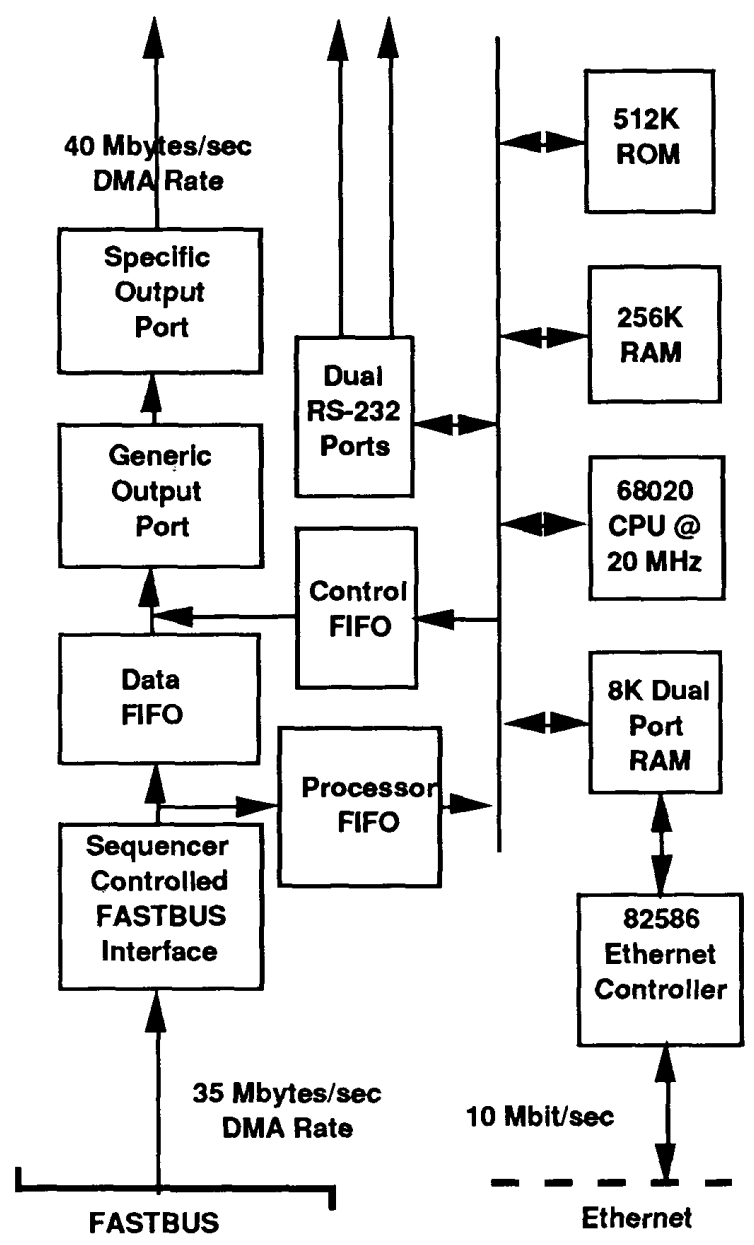

Fig. 1

The processor is a Motorola 68020 with a $20 \mathrm{MHz}$ clock. It accesses the FASTBUS through about 30 memory-mapped instructions and can access all programmable peripheral chips, including the serial line UART and INTEL 82586 Ethernet controller, through their memory mapped registers. A Multi Function Peripheral (MFP) chip is used to process all interrupts to the CPU, and enables individual masking of 8 sources of interrupts. The MFP also provides 4 programmable interval timers. The 68020 has access to 256 Kbytes of RAM and $512 \mathrm{Kbytes}$ of EPROM. In addition, the processor shares 8 Kbytes of dual ported memory with the Ethernet controller. This is used exclusively for Ethernet communications.

The processor can initiate FASTBUS block transfers to a $2 \mathrm{~K} \times 32$-bit Data FIFO. Leading word counts can be inserted between events in the Data FIFO under hardware control with no degradation in readout speed. Data can be clocked out of the
Data FIFO at $10 \mathrm{MHz}$ through the OPORT to a personality card plugged into the auxiliary backplane for a maximum output rate of $40 \mathrm{Mbytes} / \mathrm{sec}$. Event building more complicated than word count insertion requires processor intervention in data readout resulting in a much lower readout rate. As an option, data can be simultaneously written to a $256 \times$ 32-bit Processor FIFO for high speed sampling by the processor.

The FSCC can be triggered through a front panel RS-485 port. Host and terminal serial ports are available through front panel RS-232 connections. Other front panel connections are a 4 line RS-485 output port, a limo RESET line, and a thinwire Ethernet connector.

\section{SYSTEM LEVEL SOFTWARE}

Application software and device drivers are developed on VAXes using cross tools from Microtec ${ }^{3}$, Inc., including C and Pascal compilers, assembler, librarian, and linker. Source code and product management are also handled on the VAX. The Microtec Run Time Library, which supports both $C$ and Pascal, has been slightly modified to provide the process reentrancy necessary for multitasking.

A uniform embedded systems environment is used on a number of 68000 family boards in PAN-DA systems [4] (e.g., CERN GPM, Motorola MVME133A). This same environment has also been ported to the FSCC. It integrates the operating system, the board hardware, the Run Time Library, and other high level language support. The serial ports are accessible by standard language I/O constructs.

The Software Components Group operating system kemel, pSOS, and board level debugger, pROBE ${ }^{4}$, have been ported to the FSCC and are installed in PROM. All pSOS system services are directly available to applications in C or Pascal. As an aid to debugging, the environment provides a facility for extending the set of commands known to pROBE.

Applications are structured such that they may execute out of either ROM or RAM. When an FSCC is powered up or RESET, it runs an application installed in ROM. This is usually the Ethernet downloader, which can download the current target application from a Host system into RAM. The board then RESETs itself and comes up in the desired program, ensuring that the state of the board retains no history from any prior use.

${ }^{3}$ Microtec is a registered trademark of Mictrotec Research, Inc.

${ }^{4} \mathrm{pSOS}$ and pROBE are trademarks of Software Components Group, Inc. 


\section{DIAGNOSTIC SOFTWARE}

The PROM resident diagnostics are primarily targeted for initial FSCC qualification, trouble-shooting, and repair. Its menu interface is organized around the various functional blocks of the FSCC. Many menu items contain nested menus to allow tests to be customized with specific input parameters. In addition, a menu item allows many of the tests to run in an infinite loop.

The menu contains a general purpose FASTBUS diagnostic. One may selectively execute any of the FSCC FASTBUS functions. These functions may be initiated in a variety of modes, including executing single commands or a list of commands in a batch or interactive mode.

Other menu items call tests for the memory, serial port, Ethernet interface, output and trigger ports, processor and data FIFOs, and interrupt controller. Many of these tests use diagnostic supplied default values by calling the general hardware exerciser test menu option. This test is a first level confidence test for the FSCC board.

In general, the diagnostic requires additional FASTBUS hardware to exercise the FASTBUS and I/O port functions. While the FASTBUS tests may be used with any FASTBUS Slave, other tests require a FASTBUS memory module and/or a Fermilab designed FSCC Output Port test module, or a Fermilab designed FSCC front panel Trigger Port test board.

\section{COMMUNICATIONS SOFTWARE}

The FSCC is able to communicate with the outside world over RS-232 and Ethernet. The software we have layered on top of these two media is depicted in Figure 2.

Communications Software

\begin{tabular}{|c|c|c|c|}
\hline \multicolumn{2}{|c|}{$\begin{array}{l}\text { Remote } \\
\text { procedure } \\
\text { call }\end{array}$} & $\begin{array}{c}\text { Applications } \\
\text { Reliable } \\
\text { datagrams }\end{array}$ & $\begin{array}{l}\text { Unreliable } \\
\text { datagrams }\end{array}$ \\
\hline \multicolumn{2}{|c|}{ RPX } & \multirow{3}{*}{$\begin{array}{c}\text { RDS } \\
\text { Reliable } \\
\text { Datagram } \\
\text { Service }\end{array}$} & \multirow{3}{*}{$\begin{array}{l}\text { RAWPACK } \\
\text { Ethernet driver } \\
\text { and low level } \\
\text { unreliable } \\
\text { datagram } \\
\text { interface }\end{array}$} \\
\hline TRMBO & ETHBO & & \\
\hline $\begin{array}{l}\text { SPDRV } \\
\text { Serial } \\
\text { port } \\
\text { driver }\end{array}$ & & & \\
\hline & & \multicolumn{2}{|c|}{ Ethernet } \\
\hline
\end{tabular}

Fig. 2.
The Ethernet interface, in addition to providing a higher speed path to the FSCC than RS-232, reduces the number of lines required to access a data acquisition system with multiple FSCCs to one.

\section{A.Ethernet Driver}

Driver software, named RAWPACK, provides a low level, multitasking, routine interface to the Intel 82586 Ethernet controller. The routines permit processes to exchange packets with their peers over Ethernet. Since delivery to a peer is not guaranteed by the Ethernet protocol, RAWPACK only provides an unreliable datagram service (a reliable datagram package, RDS, is discussed later). A package available on the VAX, AETHER_TOOLS, provides the same routine call interface to the VMS Ethernet device driver for programming convenience.

RAWPACK software consists of user callable routines and an interrupt handler. The interrupt handler's main task is to copy incoming packets to the appropriate process based on Ethernet protocol types and, optionally, on the packet's source Ethernet address. The user callable routines allow the user to start a normal Ethernet session, start a fast transmit session, set session options, transmit a packet, receive a packet synchronously or asynchronously, and close a session.

The driver communicates with the Ethernet controller through 8 Kbytes of Dual Ported RAM and processor interrupts. A small portion of this RAM is used for control; the majority is used for system buffering of transmitted and received packets. Packets are built from chained 128 byte system buffers. The driver copies a user's buffer to or from these system buffers; system buffers are not normally accessible by user software. Sytem buffer space is split between buffers for packet reception and transmission. The division favors asynchronous reception, while allowing for up to two full sized ( $2 \times 1500$ bytes) pending transmission packets.

The packet transmission routine will not return control to the caller until it can allocate necessary resources (this behaviour can be overridden). Incoming packets are discarded if sufficient resources do not exist.

System buffers can be preallocated for "fast" transmission directly from them, bypassing most of the driver overhead. The division of system buffers was chosen to permit full size normal transmissions to take place while 1500 bytes are preallocated for fast transmissions.

A transmission rate of 770 packets $/ \mathrm{sec}$ (1.16 Mbytes/sec) for full size, 1500 byte, Ethernet packets is possible with fast transmission. This is $93 \%$ of the bandwidth of the Ethernet. The driver can perform normal full length transmissions at 637 packets/sec ( $0.96 \mathrm{Mbytes} / \mathrm{sec})$. Smaller 100 byte packet 
transmissions proceed at a rate of 5800 packets/sec for fast mode, and 2000 packets/second for normal mode.

Driver CPU overhead has been measured to be on the order of $350+(0.7 *$ NBYTES $)$ microseconds per packet, where NBYTES is the number of bytes in the (normal mode) transmitted or received packet (this figure excludes any system scheduling and process context switching overhead).

RAWPACK also includes pROBE commands to SET/SHOW local and host Ethernet addresses and to display driver statistics and data structures for diagnostic purposes.

\section{B. Serial Port Driver}

The Serial Port Driver, SPDRV, [4] was designed to provide functions for use in normal terminal dialog with the user and as a communication medium over RS-232. Its main features are:

- Fully interrupt driven: Minimizes the impact on system performance.

- Multitasking: Arbitration for multiple processes accessing a given port is automatically handled.

- Multiport access: Designed to support any number of ports on a single system.

- User control: The user has control over how a particular port operates including echoing and editing of input, line terminators, flow control, interrupting execution of the processor via a BREAK, and support for higher level language requirements.

- Optimized I/O path: A higher speed, reduced functionality data path is provided through the driver. This enables an RS-232 line to be used as a data transfer medium while minimizing the impact on system performance.

SPDRV provides a set of user callable routines to allocate and deallocate a port, read and change a port's characteristics, and read and write bytes to a port. SPDRV is well integrated into the high level embedded language environment. Therefore, applications written in Microtec $\mathrm{C}$ and Pascal have full access to SPDRV via standard language I/O features. Programs may bypass standard I/O and call the driver directly.

Output handling is done completely via interrupt processing. Since input processing is significantly more complex, it is not practical to provide all input functions during interrupt processing without seriously compromising system performance. Instead, the more complex functions such as echo and line editing are deferred to a process associated with the driver. The optimized $\mathrm{I} / \mathrm{O}$ path is an exception: input operations are serviced entirely via interrupt processing.

\section{Reliable Datagram Communications}

RDS is a set of routines which implements a Reliable Ethernet Datagram Service for the FSCC and VAX. The simplest reliable protocol, positive acknowledgement with retransmission, is used. Packets queued for transmission are sent one packet at a time and are retransmitted until an acknowledgement is received from the destination. The retransmission interval and timeouts can be changed at any time by the user. The user can also access performance statistics.

Transfer rates of $70 \mathrm{Kbytes} / \mathrm{sec}$ have been observed between a Micro-VAX II and an FSCC. This is about half the rate of the optimized TCP/IP protocol throughput to a MicroVAX II. This reduced performance is outweighed by the fact that RDS loads into 3700 bytes of memory while the Berkley BSD TCP/IP package requires about 150000 bytes - half of the available FSCC memory.

\section{Remote Procedure Execution}

In order to distribute applications among different processors and operating systems, nearly all communications within the PAN-DA data acquisition system are based on our implementation of a Remote Procedure eXecution package (RPX) [5]. It allows application tasks on VMS, pSOS, and Unix systems to communicate through subroutine calls in a standard way.

Allowing for RPX requires the simple awareness that a subroutine could execute on the same or different computer than its calling routine. By designing and implementing code with this in mind, one can code and test a package on a single processor and subsequently easily split the package, at the subroutine level, to run on multiple processors.

The RPX software has been ported to the FSCC, and as Figure 2 indicates, can be either RS-232 or Ethernet based. The software interface which provides the underlying fullduplex connection oriented protocol over RS-232 for RPX is called TRMBO. This software interface adapted for use over the Ethernet is called ETHBO, and is layered on top of RDS. RPX software needs no knowledge of which interface is in use. Ethernet based RPX is 20 times faster than RS-232 based RPX.

\section{FASTBUS STANDARD ROUTINES}

We have ported the FASTBUS Standard Routines developed at Los Alamos for the GPM [6] to run under pSOS on the FSCC. We have extended the implementation to provide a $\mathrm{C}$ calling interface and to include assembly Macros to implement a sub-set of the Standard Routines. These allow 
FASTBUS operations to be executed with minimal software overhead, while permitting use of the parameter and error reporting software built into the existing routines.

A VAX based FSCC application development environment has been created by using RPX to allow the FSCC FASTBUS Standard Routines to be called remotely from VAXes. Both E-771 and E-791 use this environment for diagnostics. E-791 also uses RPX based applications for front end readout module initialization and pedestal loading.

\section{REFERENCES}

[1] Berg, et al, "The PAN-DA Data Acquisition System", IEEE Transactions on Nuclear Science, Vol.NS-36, No.5, October 1989.

[2] See accompanying paper, "An Intelligent Readout Controller for FASTBUS, The Fermilab FSCC", Bowden et al.

[3] FASTBUS Smart Crate Controller, Design Specification, June 15 1990. Internal Fermilab document

[4] Berg, et al, "A Real Time Integrated Environment for Motorola 680xx-based VME and FASTBUS Modules" IEEE Transactions on Nuclear Science, Vol.NS-36, No.5, October 1989.

[5] E.Berman, D.Petravick, G.Sergey, "Remote Procedure Execution Software for Distributed Systems" IEEE Transactions on Nuclear Science, Vol.NS-36,No.5, October 1989

[6] Kozlowski and W.M. Foreman, "An implementation of the new IEEE Standard Routines for FASTBUS" IEEE Transactions on Nuclear Science, Page 800, Vol.NS-34, No.4, August 1987. 


\title{
A HIGH ENERGY PHYSICS RUN CONTROL SYSTEM
}

\section{BASED ON AN OBJECT ORIENTED APPROACH}

\author{
A. Masoni, G. Puddu \\ Universita' di Cagliari and INFN Sezione di Cagliari, Cagliari, Italy \\ G. Maron, G. Vedovato \\ INFN Laboratori Nazionali di Legnaro, Legnaro, Italy \\ M. Morando \\ Universita' di Padova and INFN Sezione di Padova, Padova, Italy \\ B. Minetti \\ Politecnico di Torino and INFN Sezione di Torino, Torino, Italy \\ F. D'Isep \\ INFN Sezione di Torino, Torino, Italy \\ V. Filippini \\ INFN Sezione di Pavia, Pavia, Italy
}

\begin{abstract}
The Run Control system developed for the Obelix experiment at the Low Energy Antiproton Ring of CERN is described. The adopted approach is based on a State Manager developed as a part of the MODEL project. The State Manager incorporates a model for the different activities and for the way they must be organized. An object-oriented decomposition of the on-line system is performed. A clean separation of the control, logic and operating tasks is achieved. Remote Procedure Call techniques are employed to cope with the problems of a distributed system architecture.
\end{abstract}

\section{INTRODUCTION}

Obelix [1] is a high resolution magnetic spectrometer consisting of four subdetectors covering almost the entire solid angle:

1) a Spiral Projection Chamber (SPC) used as a vertex detector;

2) jet drift chambers (AFS) for charged particle momentum and $\mathrm{dE} / \mathrm{dx}$ measurement;

3) two concentric arrays of plastic scintillators (TOF) for Time-Of-Flight measurement

4) four supermodules composed by 20 sandwiches of lead, aluminium plates and limited streamer tubes (HARGD), used for neutral particle detection.
The apparatus is presently installed at the Low Energy Antiproton Ring (LEAR) at CERN. The installation of the first three subdetectors and the related electronics is completed. For the $\gamma$ detector two supermodules are presently installed, the detector is foreseen to be fully installed by August 1991

The apparatus has taken data with this configuration in July and August 1990.

\section{SYSTEM ARCHITECTURE}

The modularity and complexity of the apparatus is reflected in the data acquisition system where a distributed architecture [2], with a high level of parallelism, has been adopted in order to cope with the large amount of raw data $(\sim 1 \mathrm{Mb})$ coming from $\sim 50000$ readout channels (see figure 1).

One of the major problems we were faced in the development of our Run Control system was the distributed nature of our apparatus and related equipments. More than 50 VME cpus and a Local Area Vax Cluster accomplish the task of collecting, formatting, recording and monitoring the data coming from the front-end electronics. This implies that many different processes running under different operating systems (OS9 for the VME and VMS for the Vax Cluster) need to be properly synchronized and executed. This is precisely the Run Control task.

The approach followed by our group is based on a State Manager (SM) [3] developed as part of the MODEL project [4]. Figure 2 shows the structure of our run control system. 